\title{
Phenotypic evaluation of the effect of anaerobiosis on some virulence attributes of Candida albicans
}

\begin{abstract}
Correspondence
Edvaldo Antonio Ribeiro Rosa edvaldo.rosa@pucpr.br
\end{abstract}

Received 7 February 2008

Accepted 4 June 2008

\author{
Edvaldo Antonio Ribeiro Rosa, ${ }^{1,2}$ Rodrigo Nunes Rached, ${ }^{1}$ \\ Sérgio Aparecido Ignácio, ${ }^{1}$ Rosimeire Takaki Rosa, ${ }^{1}$ Wander José da Silva, ${ }^{2,3}$ \\ Joyce Yick Yee Yau ${ }^{2}$ and Lakshman Perera Samaranayake ${ }^{2}$
}

${ }^{1}$ Laboratory of Stomatology, Faculty of Dentistry, Biological and Health Sciences Centre, Pontifical Catholic University of Paraná, Rua Imaculada Conceição 1155, Curitiba PR 80215-901, Brazil

${ }^{2}$ Oral Biosciences Unit, Faculty of Dentistry, Prince Philip Dental Hospital, University of Hong Kong, 34 Hospital Road, Sai Ying Pun, Hong Kong SAR

${ }^{3}$ Department of Prosthetic Dentistry, Faculty of Dentistry of Piracicaba, State University of Campinas, Avenida Limeira 901, Piracicaba SP 13414-903, Brazil

\begin{abstract}
The current assumption that Candida albicans is a facultatively anaerobic organism has been widely accepted since its recovery from anoxic sites became common. However, the link between anaerobiosis and virulence remains uncertain. This study investigated the differential cell-surface hydrophobicity (CSH) using a hydrocarbon/water partition technique and analysed the differential secretion rates of secretory aspartyl proteases (Saps), esterase, chondroitinase and haemolysins of $C$. albicans strains recovered from periodontal pockets and non-periodontium-related intra-oral sites. For the enzymic tests, all strains from both sets were grown under aerobic and anaerobic conditions and the harvested cells were inoculated onto suitable normal or pre-reduced culture media in the presence or absence of molecular oxygen, respectively. The results showed that no variations were perceptible for $\mathrm{CSH}$ and chondroitinase $(P>0.05)$. The secretion rates of esterase and haemolysins strongly decreased in an anoxic environment $(P<0.0001)$. However, a consistent increment $(P<0.0001)$ in Sap secretion was detected when cultures were grown under anaerobic conditions. Based on these results, it is suggested that the oxygen concentration in the atmosphere surrounding cells exerts a variable influence on the virulence attributes of $C$. albicans.
\end{abstract}

\section{INTRODUCTION}

The dimorphic fungus Candida albicans is the most commonly found eukaryotic micro-organism in the human oral cavity. The importance of this species in many oral mucosal diseases is well-known (McCullough et al., 1996) and most of the niches colonized by this fungus exhibit an oxygen tension and redox potential $\left(E_{\mathrm{h}}\right)$ compatible with those detected in environmental conditions favourable for the growth of aerobic or, in some instances, facultative organisms. One of the pioneering efforts to evaluate the possibility of anaerobic growth of $C$. albicans is attributed to Szawatkowski \& Hamilton-Miller (1978), who reported that aerobically cultivable strains could also grow in the complete absence of atmospheric oxygen. Nowadays, even the mating behaviour of $C$. albicans during anaerobiosis is known (Dumitru et al., 2007).

Abbreviations: $\mathrm{CSH}$, cell-surface hydrophobicity; Sap, secretory aspartyl protease.
C. albicans has the ability to regulate individual complexes and partition electron flux among the classical respiratory chain, the parallel respiratory chain and an alternative oxidase, which allows its cells to modify their energy and redox status in order to ensure survival (Ruy et al., 2006). In the complete absence of oxygen, $C$. albicans cells may also use a fermentative pathway (Ogasawara et al., 2006), which implies a reduction in cell division rates.

In recent years, interest in the lifestyle of Candida species in anoxic sites has increased. In the field of dentistry, many groups have shown that C. albicans is found in several oral niches that are not considered aerobic, such as periodontal pockets (Reynaud et al., 2001), radicular dentinal walls (Siqueira et al., 2002), infected root canals (Baumgartner et al., 2000), sites of apical periodontitis (Waltimo et al., 1997) and abscesses (McManners \& Samaranayake, 1990). However, it remains uncertain whether these yeasts contribute to the development of pathological conditions.

In general, expression of the virulence factors of C. albicans has been evaluated almost exclusively under aerobic 
conditions (i.e. environmental conditions with positive $E_{\mathrm{h}}$ values), with little investigation into the expression of fungal virulence attributes during anaerobiosis (Webster \& Odds, 1987). Only fungal pleomorphism and resistance to antimycotics have been evaluated during anaerobiosis, with abundant phenotypic transition of yeast to hyphae being described when C. albicans is grown under strict anaerobiosis (Dahle \& Olsen, 1991; Kaminishi et al., 1994). Dumitru et al. (2004) found that C. albicans grown under anaerobiosis tended to become more resistant to azoles and polyenes than the same yeast strain grown under aerobic conditions.

Because C. albicans is found in intraoral tissues and niches under anaerobic conditions, it is plausible that this fungus may play a role in pathological conditions associated with this type of environment. Thereby, in order to predict the extent of this role, this study aimed to evaluate the effect of oxidoreductive environmental conditions on expression of virulence factors of oral C. albicans.

\section{METHODS}

C. albicans strains. Strains $3 \mathrm{~A} 1,13 \mathrm{~A} 3,14 \mathrm{~A} 3,15 \mathrm{~A} 2,30 \mathrm{~A} 2,30 \mathrm{~A} 4$, 47A4, 55A3, 57A1 and 58A4 were recovered from periodontal pockets with a depth equal to or greater than $5 \mathrm{~mm}$ and were kindly provided by Professor José F. Höfling (Faculty of Dentistry of Piracicaba, Brazil). Strains B3, B8, C1, F1, F2, N1, N2, N3 and SC5314 were kindly provided by Dr Thein Zaw Moe (Oral Biosciences Unit, University of Hong Kong, Hong Kong) and were of diverse origins not related to periodontal disease. Strain ATCC 90028 was used as a reference strain.

Normal and reduced culture media. The culture broths used were YPD (2\% dextrose, $2 \%$ peptone, $10 \%$ yeast extract) and rYPD (YPD plus $0.1 \%$ L-cysteine, $\mathrm{pH} 6)$. The semi-solid culture media used were BSAA ( $1 \%$ yeast extract, $2 \%$ glucose, $1.5 \%$ agar, $0.2 \%$ BSA fraction V, $0.25 \%$ Protovit Plus, $\mathrm{pH}$ 4), rBSAA (BSSA plus $0.1 \%$ L-cysteine), T80A ( $1 \%$ peptone, $0.5 \% \mathrm{NaCl}, 0.1 \% \mathrm{CaCl}_{2}, 1.5 \%$ agar, $1 \%$ Tween $80, \mathrm{pH} 7.0$ ), rT80A (T80A plus $0.1 \%$ L-cysteine), CSA ( $1 \%$ yeast extract, $2 \%$ glucose, $1.5 \%$ agar, $1 \%$ chondroitin sulfate, $0.05 \%$ BSA fraction $\mathrm{V}, \mathrm{pH} 7.0$ ), rCSA (CSA plus $0.1 \%$ L-cysteine) and blood agar (3\% glucose, $1 \%$ agar, $7 \%$ sheep blood, $\mathrm{pH} 5.5$ ).

Preparation of aerobic and anaerobic inocula. C. albicans cells were grown aerobically in $3 \mathrm{ml}$ YPD at $37^{\circ} \mathrm{C}$. The culture was monitored until it reached an $\mathrm{OD}_{660}$ of 0.55 (Beckman DU 530 UV/VIS Spectrophotometer). The cells were then harvested, washed three times in $50 \mathrm{mM}$ PBS (pH 6.5) and stored at $4{ }^{\circ} \mathrm{C}$ in $3 \mathrm{ml}$ of the same buffer. This culture was designated the aerobic inoculum of C. albicans.

The following anaerobic cultures and manipulations were carried out in an anaerobic chamber (Coy Laboratory Products) with $85 \% \mathrm{~N}_{2}$, $10 \% \mathrm{CO}_{2}$ and $5 \% \mathrm{H}_{2}$. One hundred microlitres of aerobic culture was used to inoculate rYPD tubes. The cultures were incubated under anaerobic conditions and agitated at 150 r.p.m. at $37^{\circ} \mathrm{C}$. After growth, aliquots of $100 \mu \mathrm{l}$ were taken and used to inoculate another set of rYPD tubes, which were incubated anaerobically at $37{ }^{\circ} \mathrm{C}$ with agitation at 150 r.p.m. until they achieved the optical density of a 7 MacFarland standard. These two-step inoculations were necessary to adapt the $C$. albicans strains to an anaerobic environment. The cells were washed twice with $0.1 \%$ L-cysteine $/ 0.9 \% \mathrm{NaCl}$ solution in sealed tubes and finally suspended in the washing solution at an $\mathrm{OD}_{660}$ of 0.55 .
Semi-quantification of extracellular enzymes and haemolysins. Plates with normal and reduced semi-solid culture media as described above were inoculated with four $5 \mu \mathrm{l}$ spots of aerobic or anaerobic suspension onto the surface and left to dry on a laboratory bench or inside an anaerobic chamber, respectively. After incubation at $37{ }^{\circ} \mathrm{C}$ in a normal or anaerobic atmosphere for 10 days, the diameter of the hyaline zone around the colonies was measured using a digital caliper (UPM) with a resolution of $0.01 \mathrm{~mm}$ and an accuracy of $\pm 0.02 \mathrm{~mm}$. Blood agar plates were evaluated at $48 \mathrm{~h}$ post-inoculation. Halo diameter measurements were carried out following the cardinal orientations $\mathrm{N} \rightarrow \mathrm{S}, \mathrm{E} \rightarrow \mathrm{W}, \mathrm{NE} \rightarrow \mathrm{SW}$ and $\mathrm{NW} \rightarrow \mathrm{SE}$ and mean values were used for statistical analysis. Each experiment was carried out in duplicate.

Cell-surface hydrophobicity (CSH). The CSH of C. albicans cells was measured using the method described by Sweet et al. (1987). Aerobic cells were grown on YPD for $24 \mathrm{~h}$ in a normal oxygen atmosphere at $37{ }^{\circ} \mathrm{C}$. Anaerobic cells were grown on rYPD for 10 days in an anaerobic atmosphere at $37^{\circ} \mathrm{C}$. A loopful of each culture was centrifuged and washed twice with PBS ( $\mathrm{pH} 7.0)$ and suspended in PBS at an $\mathrm{OD}_{520}$ of $1.0 \pm 0.2$.

For each organism/atmospheric condition tested, $5 \mathrm{ml}$ suspension was added to four glass test tubes, representing three tests and one control. Within a fume hood, $1 \mathrm{ml}$ xylene (pro-analysis grade) was added to each test suspension. The tests and control tubes were placed in a water bath at $37^{\circ} \mathrm{C}$ for $10 \mathrm{~min}$ to equilibrate. They were then vortexed for $30 \mathrm{~s}$ and returned to the water bath for a further $30 \mathrm{~min}$ to allow the immiscible xylene and aqueous phases to separate. The lower aqueous phases of the samples were removed carefully using a pipette and transferred to clean polystyrene tubes. Any traces of contaminating xylene carried over in the pipette or bound to the yeast were removed by attaching to the tube walls. The $\mathrm{OD}_{520}$ was measured as above.

Hydrophobicity values were expressed as the percentage reduction in optical density of the test suspension compared with the control. Thus the greater the change in optical density, the greater the shift in yeast cells from the bulk medium to the interface, i.e. the more hydrophobic the yeast isolate. Each assay was conducted in triplicate on two separate occasions.

Statistics. All experimental steps above were repeated in quadruplicate, on two different occasions, to give eight separate results. All data were evaluated in relation to their normal distribution by a Kolmogorov-Smirnov test and in relation to their homogeneities of variances by the Levene index. Differences were analysed using a Mann-Whitney U-test using the statistical package SPSS 13.0. A $P$ value of 0.05 was assumed to be statistically significant.

\section{RESULTS AND DISCUSSION}

Enzymic and haemolytic activities (so-called $\mathrm{Pz}$ values) were determined by the ratio of the diameter of the colony to the total diameter of the colony plus the digestion/ precipitation zone (Samaranayake et al., 1984). Thus, a $\mathrm{Pz}$ value of 1 indicates no activity, whilst $\mathrm{Pz}<1$ indicates the degree of enzymic positivity. In order to facilitate the interpretation of our results, we used the value $1-\mathrm{Pz}$, so that the numeric results became greater as the enzymic activity increased.

All 20 C. albicans strains required $18-24$ h to achieve an $\mathrm{OD}_{660}$ of $0.516-0.548$ under aerobiosis. However, it was necessary to wait $72 \mathrm{~h}$ and a further $96 \mathrm{~h}$ to achieve the 
same cell density after the first and second anaerobic inoculations, respectively.

Compiled data concerning the enzymic activities are presented in Fig. 1. All strains, independent of their origin or the atmospheric environment, secreted secretory aspartyl proteases (Saps). Non-parametric comparisons among the subgroups revealed that, under anaerobic conditions, candidal cells tended to secrete significantly higher amounts of Saps than under aerobic conditions $(P$ $<0.0001)$. Saps are considered important molecules for the invasion and destabilization of tissues (Felk et al., 2002; Hube et al., 1997; Naglik et al., 2003) and their secretion rates increase significantly when grown under anoxic conditions. Transcription of the genes SAP4, SAP5 and SAP6 is either up- or downregulated by the transcription factors Efg1 and Cph1 depending on environmental signals such as pH or temperature (Felk et al., 2002). These two transcription factors are also directly involved in hyphal development (Biswas et al., 2007). We postulate that they may also be implicated in the anaerobic overexpression of Saps, once enough data have been gathered supporting the growth of C. albicans as hyphae in this type of environment (Dahle \& Olsen, 1991; Kaminishi et al., 1994). Further studies involving SAP4-SAP6 and other genes of the Sap family will elucidate this.

However, it cannot be generalized that anaerobiosis pushes the yeast to become more virulent, following a decrease in the secretion rates of other important factors such as haemolysins and esterase $(P<0.0001$ for both $)$. In the particular case of haemolysins, Luo et al. (2001) showed that glucose added to aerobic blood agar can increase the detection of haemolytic activity in some strains. Shuster et al. (2004) described the phenomenon of 'microbial alcohol-conferred haemolysis' and showed that ethanol is readily oxidized to acetaldehyde with a strong haemolytic behaviour. Shuster et al. (2004) also found that this phenomenon is not observed when cells are grown under anaerobiosis. However, in a subsequent study, Shuster et al. (2007) presented a new premise based on forced overloading on the respiratory mode required to oxidize vast amounts of alcohol. This effect resulted in the production of high levels of hydrogen peroxide, a well-recognized haemolytic agent. Our results showed that all strains grown in the presence of oxygen secreted haemolytic products, whereas only a few (7/20) had this phenotype during anaerobiosis (Fig. 1). As we added glucose to the culture medium, it is reasonable that these few strains anaerobically fermented this substrate to ethanol, which, in turn, was readily oxidized to acetaldehyde (Shuster et al., 2004), or had their alcohol dehydrogenase genes switched off, arresting the fermentative process at the aldehyde ending point. On plates incubated under normal levels of oxygen, pyruvate enters the tricarboxylic acid cycle and the electron-transport chain, driving all strains towards hydrogen peroxide production. We believe that enhanced haemolysis may not be attributed to anaerobiosis per se, as it was recently shown that blood cells can be stored better
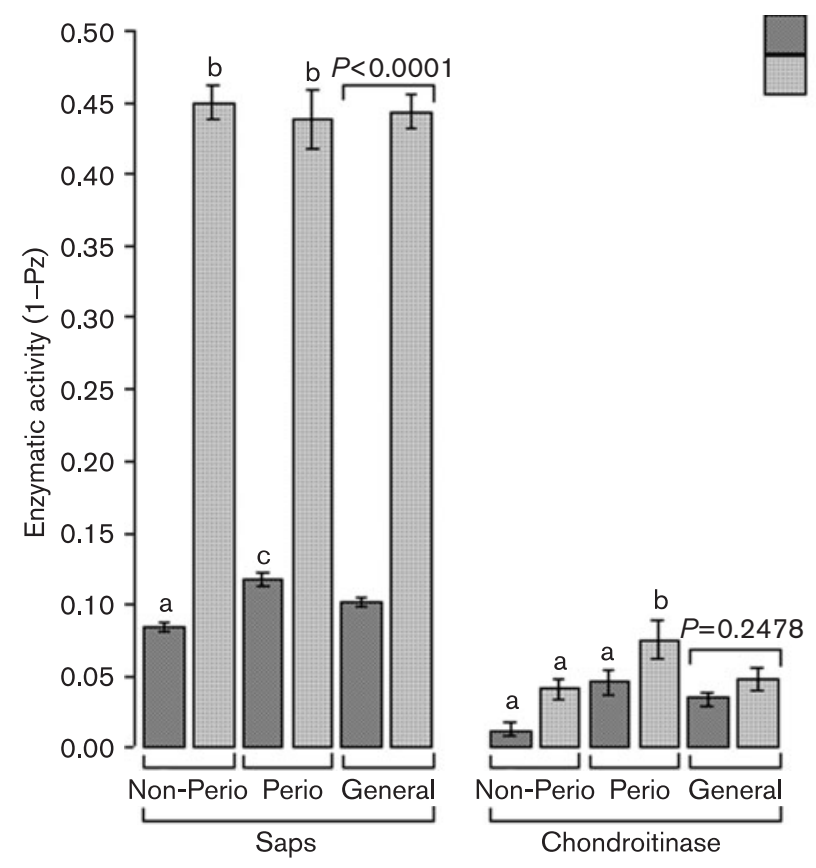

Aerobiosis

Anaerobiosis

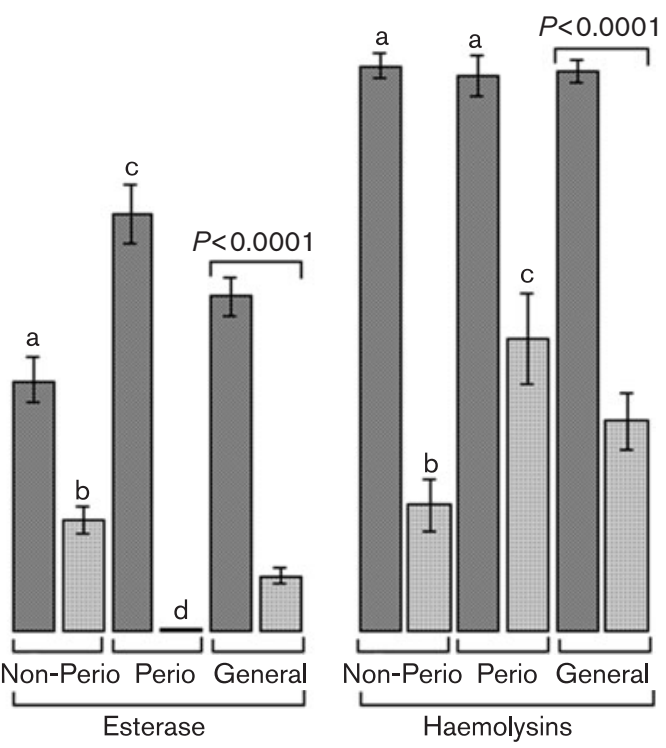

Fig. 1. Differential activity of Saps, chondroitinase, esterase and haemolysins secreted by periodontium-related (Perio) and non-periodontium-related (Non-Perio) C. albicans strains grown under aerobiosis and anaerobiosis. Results are given as means \pm SEM. Different letters denote $P$ values less than 0.05 . General data were obtained by the sum of results for periodontium-related and non-periodontium-related strains. 
under such environmental conditions rather than under conditions of normal oxygen levels (Kriebardis et al., 2007; Yoshida et al., 2007).

Hube et al. (2000) studied the lipolytic behaviour of strain SC5314 in Tween 40 and found simultaneous expression of lipase and proteinase genes. Our results with cells growing in the absence of oxygen contrast with this, as, once the expression of Saps was increased, the esterase/lipase enzymes showed a diminished rate of secretion ( $P$ $<0.0001$ ) or were completely inhibited (Fig. 1). It is likely that the anaerobic conditions alter the normal relationship between these two classes of enzyme. Hube et al. (2000) also showed that some lipase genes are always expressed during the yeast-to-hyphal transition under aerobic conditions. Again, our results revealed that the absence of oxygen generates a peculiar behaviour. Regardless of the fact that cells growing under anaerobiosis tend to grow as mycelia (Dahle \& Olsen, 1991; Kaminishi et al., 1994), the secretion of esterase exhibited a more divergent pattern than that expected for aerobiosis. Our belief is that the anaerobic conditions or the reductive environment guide lipolytic enzyme production towards alternative pathways.

Interesting, four periodontium-related strains showed no enzyme secretion when grown under anaerobiosis (data not shown). This finding corroborates the observations of Kurnatowska (1998), who showed that strains isolated from gingivitis and juvenile/adult periodontitis did not secrete, or only secreted discrete amounts of esterase, whilst strains isolated from mucous infection sites secreted a higher level of esterase. Therefore, it is possible that the periodontal pocket environment selects C. albicans strains whose esterase expression is inhibited in the absence of molecular oxygen.

The secretion of chondroitinase was not constant and only three strains secreted this enzyme. The atmospheric conditions did not exert much influence on the chondroitinase secretion phenotype once no differences were assessed for the summed data $(P=0.2478)$, although a discrete elevation in enzymic activity was observed when strains were grown in the absence of oxygen (Fig. 1). Few studies have shown the virulence potential of this enzyme in fungi (Coutinho \& Paula, 2000; de Assis et al., 2003; Shimizu et al., 1995), and to our knowledge no data concerning gene expression, signal transduction or biochemical aspects of this enzyme in fungi are available.

Fig. 2 shows that $\mathrm{CSH}$ differences were not significant $(P=0.9010)$ except for the non-periodontium-related strains submitted to anoxia, whose mean value was less than that of the others $(P<0.0001)$. We suppose that, as such strains are adapted to grow in the presence of oxygen, when shifted to anoxic conditions their cell walls could not rearrange the hydrophobic proteins properly. However, this raises the question of the importance of this $\mathrm{CSH}$ reduction from $75.78-78.39 \%$ to $68.62 \%$. Although statistically significant, this reduction did not appear to confer a less virulent phenotype on these strains.

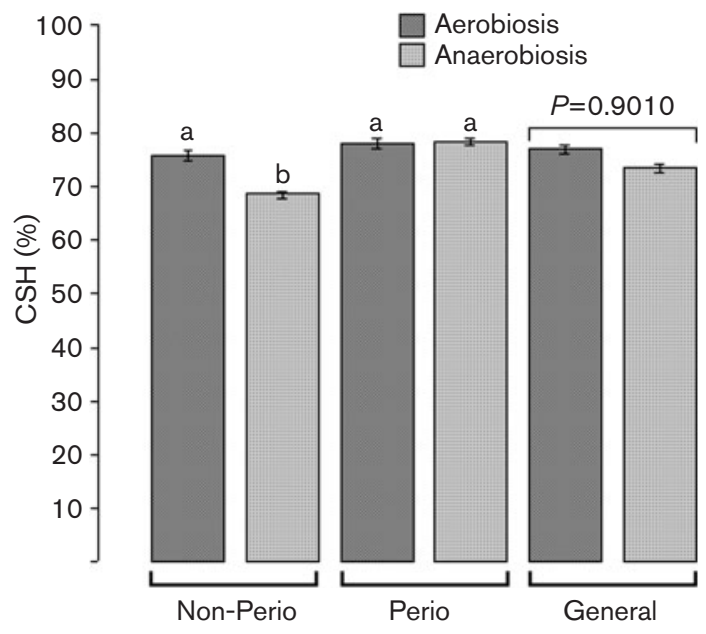

Fig. 2. Differential CSH of periodontium-related and non-periodontium-related C. albicans strains grown under aerobiosis and anaerobiosis. Results are given as means \pm SEM. Different letters denote $P$ values less than 0.05 . General data were obtained by the sum of results for periodontium-related and non-periodontiumrelated strains.

In conclusion, according to the results obtained in this study, it is clear that different atmospheric/redox conditions can exert a considerable influence over the virulence attributes of C. albicans.

\section{ACKNOWLEDGEMENTS}

The authors are indebted to Professor José F. Höfling (FOPUNICAMP) and Dr Thein Zaw Moe (OBU-PPDH-HKU) for the yeast strains used in this study. This work was supported by grants supplied by the Araucaria Foundation (Process 9042) and CAPES Coordination for the Improvement of Higher Education Personnel (Process BEX 0559/06-7) and intramural funds from OBU-PPDHHKU.

\section{REFERENCES}

Baumgartner, J. C., Watts, C. M. \& Xia, T. (2000). Occurrence of Candida albicans in infections of endodontic origin. J Endod 26, 695-698.

Biswas, S., Van Dijck, P. \& Datta, A. (2007). Environmental sensing and signal transduction pathways regulating morphopathogenic determinants of Candida albicans. Microbiol Mol Biol Rev 71, 348-376.

Coutinho, S. D. \& Paula, C. R. (2000). Proteinase, phospholipase, hyaluronidase and chondroitin-sulfatase production by Malassezia pachydermatis. Med Mycol 38, 73-76.

Dahle, U. R. \& Olsen, I. (1991). Anaerobiosis and serum promote mycelium formation by Candida albicans in colonies on TSBV agar. Acta Odontol Scand 49, 41-45.

de Assis, C. M., Gandra, R. F., Gambale, W., Shimizu, M. T. \& Paula, C. R. (2003). Biosynthesis of chondroitinase and hyaluronidase by different strains of Paracoccidioides brasiliensis. J Med Microbiol 52, 479-481. 
Dumitru, R., Hornby, J. M. \& Nickerson, K. W. (2004). Defined anaerobic growth medium for studying Candida albicans basic biology and resistance to eight antifungal drugs. Antimicrob Agents Chemother 48, 2350-2354.

Dumitru, R., Navarathna, D. H., Semighini, C. P., Elowsky, C. G., Dumitru, R. V., Dignard, D., Whiteway, M., Atkin, A. L. \& Nickerson, K. W. (2007). In vivo and in vitro anaerobic mating in Candida albicans. Eukaryot Cell 6, 465-472.

Felk, A., Kretschmar, M., Albrecht, A., Schaller, M., Beinhauer, S., Nichterlein, T., Sanglard, D., Korting, H. C., Schäfer, W. \& other authors (2002). Candida albicans hyphal formation and the expression of the Efg1-regulated proteinases Sap4 to Sap6 are required for the invasion of parenchymal organs. Infect Immun 70, 3689-3700.

Hube, B., Sanglard, D., Odds, F. C., Hess, D., Monod, M., Schäfer, W., Brown, A. J. \& Gow, N. A. (1997). Disruption of each of the secreted aspartyl proteinase genes SAP1, SAP2, and SAP3 of Candida albicans attenuates virulence. Infect Immun 65, 3529-3538.

Hube, B., Stehr, F., Bossenz, M., Mazur, A., Kretschmar, M. \& Schäfer, W. (2000). Secreted lipases of Candida albicans: cloning, characterization and expression analysis of a new gene family with at least ten members. Arch Microbiol 174, 362-374.

Kaminishi, H., Iwata, A., Tamaki, T., Cho, T. \& Hagihara, Y. (1994). Spiral hyphae of Candida albicans formed in anaerobic culture. Mycoses 37, 349-352.

Kriebardis, A. G., Antonelou, M. H., Stamoulis, K. E., EconomouPetersen, E., Margaritis, L. H. \& Papassideri, I. S. (2007). Storagedependent remodeling of the red blood cell membrane is associated with increased immunoglobulin G binding, lipid raft rearrangement, and caspase activation. Transfusion 47, 1212-1220.

Kurnatowska, A. J. (1998). Activity of hydrolytic enzymes of Candida albicans strains isolated from patients with periodontal and membrane mucosa of oral cavity diseases. Mycopathologia 141, 105-109.

Luo, G., Samaranayake, L. P. \& Yau, J. Y. (2001). Candida species exhibit differential in vitro hemolytic activities. J Clin Microbiol 39, 2971-2974.

McCullough, M. J., Ross, B. C. \& Reade, P. C. (1996). Candida albicans: a review of its history, taxonomy, epidemiology, virulence attributes, and methods of strain differentiation. Int J Oral Maxillofac Surg 25, 136-144

McManners, J. \& Samaranayake, L. P. (1990). Suppurative oral candidosis. Review of the literature and report of a case. Int J Oral Maxillofac Surg 19, 257-259.
Naglik, J. R., Challacombe, S. J. \& Hube, B. (2003). Candida albicans secreted aspartyl proteinases in virulence and pathogenesis. Microbiol Mol Biol Rev 67, 400-428.

Ogasawara, A., Odahara, K., Toume, M., Watanabe, T., Mikami, T. \& Matsumoto, T. (2006). Change in the respiration system of Candida albicans in the lag and log growth phase. Biol Pharm Bull 29, 448-450.

Reynaud, A. H., Nygaard-Ostby, B., Boygard, G. K., Eribe, E. R., Olsen, I. \& Gjermo, P. (2001). Yeasts in periodontal pockets. J Clin Periodontol 28, 860-864.

Ruy, F., Vercesi, A. E. \& Kowaltowski, A. J. (2006). Inhibition of specific electron transport pathways leads to oxidative stress and decreased Candida albicans proliferation. J Bioenerg Biomembr 38, 129-135.

Samaranayake, L. P., Raeside, J. M. \& MacFarlane, T. W. (1984). Factors affecting the phospholipase activity of Candida species in vitro. Sabouraudia 22, 201-207.

Shimizu, M. T., Jorge, A. O., Unterkircher, C. S., Fantinato, V. \& Paula, C. R. (1995). Hyaluronidase and chondroitin sulfatase production by different species of Candida. J Med Vet Mycol 33, 27-31.

Shuster, A., Osherov, N. \& Rosenberg, M. (2004). Alcohol-mediated haemolysis in yeast. Yeast 21, 1335-1342.

Shuster, A., Osherov, N., Leikin-Frenkel, A. \& Rosenberg, M. (2007). Alcohol-conferred hemolysis in yeast is a consequence of increased respiratory burden. FEMS Yeast Res 7, 879-886.

Siqueira, J. F., Jr, Rocas, I. N., Lopes, H. P., Elias, C. N. \& de Uzeda, M. (2002). Fungal infection of the radicular dentin. J Endod 28, 770-773.

Sweet, S. P., MacFarlane, T. W. \& Samaranayake, L. P. (1990). An in vitro method to study the adherence of bacteria to saliva-treated tooth enamel sections. Oral Microbiol Immunol 5, 24-28.

Szawatkowski, M. \& Hamilton-Miller, J. M. T. (1978). Anaerobic growth and sensitivity of Candida albicans. Microbios Lett 5, 61-66.

Waltimo, T. M., Siren, E. K., Torkko, H. L., Olsen, I. \& Haapasalo, M. P. (1997). Fungi in therapy-resistant apical periodontitis. Int Endod J 30, 96-101.

Webster, C. E. \& Odds, F. C. (1987). Growth of pathogenic Candida isolates anaerobically and under elevated concentrations of $\mathrm{CO}_{2}$ in air. J Med Vet Mycol 25, 47-53.

Yoshida, T., AuBuchon, J. P., Tryzelaar, L., Foster, K. Y. \& Bitensky, M. W. (2007). Extended storage of red blood cells under anaerobic conditions. Vox Sang 92, 22-31. 